\title{
Las citas bibliográficas del IIMAS en el Web of Science (ISI), Scopus (Elsevier) y Google Académico (Google): ¿diferentes o semejantes?
}

Cited works of the Instituto de Investigaciones en Matemáticas Aplicadas y en Sistemas compared in Web of Science, Scopus (Elsevier) and Google Scholar databases: differences and resemblances

\section{Suyin Ortega Cuevas}

Instituto de Investigaciones en Matemáticas Aplicadas y en Sistemas. Universidad Nacional Autónoma de México. México D. F. CP. 04510. suyin@leibniz.iimas.unam.mx

\section{Resumen}

Se realiza un análisis comparativo de las citas bibliográficas hechas a los artículos en revistas por parte de los investigadores adscritos al Instituto de Investigaciones en Matemáticas Aplicadas y en Sistemas (IIMAS) en Web of Science, Scopus y Google Académico.

Palabras clave: Análisis de citas. Web of science. Scopus. Google Académico.

\section{Introducción}

El Instituto de Investigaciones en Matemáticas Aplicadas y en Sistemas (IIMAS) es una dependencia de la Universidad Nacional Autónoma de México (UNAM) y tiene el objetivo de "Realizar investigación científica original en las disciplinas que se cultivan en el Instituto" (Informe de actividades 2006).

Para tal fin cuenta con 94 académicos distribuidos en seis departamentos: Matemáticas y Mecánica, Métodos Matemáticos y Numéricos, Modelación Matemática en Sistemas Sociales, Probabilidad y Estadística, Ciencia de la Computación e Ingeniería en Sistemas Computacionales y Automatización.

Los investigadores realizan investigación original en las áreas correspondientes, por lo que la Biblioteca del Instituto apoya estas actividades mediante los servicios convencionales y los diseñados por el área de Servicios Especializados a través de la cual se brindan los servicios de alerta, diseminación selectiva de información, boletines de nuevas adquisiciónes y el análisis de citas bibliográficas.

\begin{abstract}
A comparative citation analysis of the publications of the researchers of the Instituto de Investigaciones en Matemáticas Aplicadas y en Sistemas (IIMAS) in the Web of Science, Scopus and Google Academic.

Keywords: Citation analysis. Web of science. Scopus. Scholar Google.
\end{abstract}

Las citas bibliográficas es un tema importante dentro del mundo de la investigación, el conocer ¿quién ha consultado un artículo? ¿Cuántas veces ha sido citado y por quién? Es motivación para los estudiosos del tema, pero surge siempre una pregunta: ¿en donde localizar estas citas? ¿Quién más esta tratando el mismo tema? ¿En que parte del mundo se estudia lo que analiza un determinado investigador? ¿Quién consulta lo que escribe? ¿Dónde localizar esas citas? Esto es una preocupación para los que se dedican a realizar estudios de investigación.

Bajo este panorama, en el presente trabajo se realizó un análisis comparativo de las citas bibliográficas que proporcionan estas bases de datos y realizó una evaluación de los sitios Web para tener una visión más amplia sobre estos servicios.

\section{La Biblioteca}

La Biblioteca-IIMAS tiene como objetivo principal "satisfacer las necesidades de información del personal académico del Instituto, de los profesores y estudiantes de los posgrados en que participa el IIMAS y en general a la comunidad universitaria, en las áreas del conocimiento que 
se cultivan en el Instituto" (Informe de actividades 2006).

Las áreas que conforman la Biblioteca IIMAS son: la Jefatura, el área de Servicios al Público (préstamo en sala, a domicilio, interbibliotecario, fotocopiado, localización de documentos, entre otros), el área de Servicios Técnicos (adquisición de libros, suscripción de revistas, encuadernación, entre otras actividades) y el área de Servicios Especializados (servicios de alerta, diseminación selectiva de información, boletines de nuevas adquisiciones, elaboración de los perfiles de interés, análisis de citas bibliográficas).

Se hablará del área de Servicios Especializados, ya que es la que se dedica a localizar las citas de los investigadores. Este servicio es exclusivamente interno, cuando ha sido solicitado por investigadores externos, estos son remitidos a la Dirección General de Bibliotecas (DGB) de la UNAM, en donde se brinda el servicio a quién lo solicite mediante un costo.

Hasta el año de 2003 el servicio de análisis de citas era una actividad realizada por el área de Servicios al Público. Sin embargo, debido a la profundidad que el análisis de citas al autor requiere y a la experiencia en la sistematización de la búsqueda, la Jefatura de la Biblioteca decide asignarla al área de Servicios Especializados.

Al establecerse el servicio solamente se realizaba el análisis de citas en el Web of Science. Su cobertura en línea es de 1995 a la fecha. Para consultar años retrospectivos, se acude a la Dirección General de Bibliotecas (DGB) para los años correspondientes de 1969 a 1994.

De esta forma, el área de Servicios Especializados presenta resultados a los investigadores, quienes al revisarlos comienzan a preguntar dónde localizan más citas, ya que saben que no son todas las reportadas. Los investigadores también solicitan que se busquen citas a trabajos de otros autores, para ver lo que están escribiendo y de esta manera observan quien escribe sobre el mismo tema.

Para ese entonces solamente se realizaba la búsqueda de información en el Web of Science. Sin embargo, ante la demanda de los investigadores de localizar más citas, se empiezan a explorar otras fuentes de información.

A partir del año de 2004 , se incrementó la demanda de solicitudes por parte de los investigadores del IIMAS y al mismo tiempo aumentó la necesidad de localizar más citas. Por ello, se buscaron otras alternativas para complementar este trabajo. En ese año la empresa Elsevier llevó a la UNAM su nuevo producto Scopus y le otorga una cuenta de prueba a la UNAM, con la que tiene acceso los artículos en revistas $y$, como valor agregado, aporta citas de los artículos que contiene su base de datos, haciendo posible la comparación de sus resultados con los proporcionados por el Web of Science.

En Internet aparece un buscador llamado Google Académico, perteneciente a Google que brinda información académica de artículos de revistas y capítulos de libros, en la mayoría de los casos con citas disponibles que permiten realizar su análisis.

Por lo anterior, se comienzan a buscar citas utilizando las bases de datos Scopus y Google Académico, pero al hacerlo, se triplica el trabajo, ya que se debe tener cuidado de no duplicar o triplicar las citas, lo que daría una impresión negativa.

\section{Los buscadores}

Para facilitar información, primeramente se debe de tener una visión general del sitio donde se obtendrá y saber qué es lo que proporciona y la confiabilidad del mismo. Se consideró conveniente evaluar las bases de datos Web of Science, Scopus y Google Académico con base en los parámetros del artículo titulado Evaluación de recursos digitales en línea: conceptos, indicadores y métodos (Codina, 2000).

Las tres bases de datos tienen una manera muy particular de mostrar los registros bibliográficos. Para ello, se describe brevemente cada una. Es importante mencionar que las dos primeras tienen un costo por suscripción y la última es de acceso libre.

\subsection{Web of Science}

Science Citation Index, producido por la empresa ISI Thomson desde el año de 1960, había sido el único productor de este tipo de bases de datos.

EI ISI indiza anualmente cerca de 8.700 revistas de alto impacto. Para seleccionar si una revista es incluida o no dentro de esta base de datos toma en cuenta los siguientes criterios: puntualidad en su frecuencia; título, resumen, palabras clave en inglés, adicionales a su idioma original; contenido editorial acorde con la disciplina de la revista; diversidad internacional (incluir revistas de diversas regiones para hacerlo multinacional); y debe ser una revista impresa, aunque ya considera algunas revistas que solamente se editan en formato electrónico. 


\subsection{Scopus}

Es producida por la empresa Elsevier a partir de 2004. Esta base contiene referencias bibliográficas aportando también el servicio de citas; Scopus lo conforman aproximadamente 15.000 títulos de revistas y los criterios de inclusión son los siguientes: deben incluir el Internacional Estándar Serial Number (ISSN); ser revistas de publicación regular; deben ser artículos de tipo documental; considera revistas de todo el mundo; comprende revistas a partir de 2004, aunque conjunta registros de otras bases de datos, por ejemplo, Medline, que contiene registros a partir de 1966 y de algunas otras más para obtener información retrospectiva; y contiene además de artículos, páginas Web y patentes, entre otros.

\subsection{Google Académico}

Google Académico surge en 1998 como una iniciativa de un grupo de personas que buscan un método de búsqueda por Internet rápido y fácil. Este sitio Web, además de ser gratuito, no tiene limitaciones en el tipo de material, que puede ser artículo en revista, libro o tesis y, en algunos casos, hasta el texto completo. Como información adicional proporciona las citas bibliográficas en la mayoría de los artículos que contiene. El único criterio de inclusión es ser una página Web. Para efectos del presente trabajo la información mostrada de las tres bases es suficiente para obtener una perspectiva de lo que ofrecen, por lo que ahora se evaluará según la adaptación que se hizo de los parámetros que proporciona Codina.

\section{Metodología}

Primeramente, se analizaron los indicadores propuestos por Codina, para determinar los parámetros que se utilizarían para realizar la evaluación. Se analizó cada uno, y solamente se contemplaron los que proporcionaban una visión global de los resultados a obtener.

La evaluación se realiza bajo dos esquemas: micronavegación y macronavegación. La micronavegación se refiere a la manera de presentar la información, e incluye la calidad y volumen de la información, la autoría, la legibilidad y ergonomía (facilidad de lectura, buen diseño y que no canse la vista), la navegación y representación de la información y la recuperabilidad. La macronavegación se refiere a las características que tiene hacia fuera de la página, esto es, la luminosidad, la calidad de la información, y la actualización de ligas.

Posteriormente se realizó la evaluación dando a cada reactivo un símbolo de presencia de la información asignándole $(\sqrt{ })$, en caso contrario se le fijó $(X)$ y si la información no es clara se colocó (?). La evaluación que se realizó fue de tipo cualitativa y subjetiva, considerando que no es pertinente dar valores numéricos pues representa mejor la valoración con ausencia o presencia de los indicadores.

Finalmente se efectuaron varias estrategias de búsqueda sobre un mismo nombre y las diferentes variantes que puede tener, para analizar los resultados que presentan las tres bases de datos. Se aplica la misma estrategia para observar que tan similares o diferentes son los datos que proporcionan y de esta manera llegar a una conclusión privilegiada.

\section{Resultados}

Con base a los indicadores propuestos por Codina, se realizó la evaluación de las tres bases de datos, obteniendo los resultados siguientes.

\subsection{Micronavegación}

La tabla I se refiere a la calidad y volumen de la información, Google Académico fue el único que no muestra una consistencia en la presentación de los resultados, ya que en lo que concierne a rigor la información no está tratada con cuidado, no tiene una cobertura de todo el tema careciendo de criterios de selección y de edición. No contiene los datos propios de una referencia bibliográfica.

\begin{tabular}{lccc}
\hline & $\begin{array}{c}\text { Web of } \\
\text { Science }\end{array}$ & Scopus & $\begin{array}{c}\text { Google Aca- } \\
\text { démico }\end{array}$ \\
\hline Rigor & $\sqrt{ }$ & $\sqrt{ }$ & $\mathrm{X}$ \\
\hline Volumen & $\sqrt{ }$ & $\sqrt{ }$ & $\mathrm{X}$ \\
\hline Edición & $\sqrt{ }$ & $\sqrt{ }$ & $\mathrm{X}$ \\
\hline
\end{tabular}

Tabla I. Calidad y volumen de la información.

Las tres bases de datos (Tabla III) especifican claramente la mención de autoría de las empresas que las editan, incluyendo datos de contacto. Este es un punto adecuado pues refleja la seriedad de los autores para hacerse responsables de la información.

\begin{tabular}{lccc}
\hline & $\begin{array}{c}\text { Web of } \\
\text { science }\end{array}$ & Scopus & $\begin{array}{c}\text { Google } \\
\text { Académico }\end{array}$ \\
\hline Responsable & $\sqrt{ }$ & $\sqrt{ }$ & $\sqrt{ }$ \\
\hline $\begin{array}{l}\text { Reconocimiento } \\
\text { en la especiali- } \\
\text { dad }\end{array}$ & $\sqrt{ }$ & $\sqrt{ }$ & $\sqrt{ }$ \\
\hline
\end{tabular}

Tabla II. Autoría. 
La legibilidad y la ergonomía es un punto verdaderamente importante, ya que de nada sirve que la información sea buena si no se puede visualizar adecuadamente. De las tres bases de datos, Google Académico no cumple totalmente este indicador, puesto que no edita la información. Al momento de mostrar los resultados, hay algunos que no son fáciles de leer, ya sea por la tipografía o por los colores que contiene la información de origen. En cambio, Scopus y Web of Science hacen captura original de los resultados: todos tienen una uniformidad, y esto permite que la legibilidad y la ergonomía sea buena ante los ojos del usuario. Estos resultados se visualizan en la tabla III.

\begin{tabular}{lccc}
\hline & $\begin{array}{c}\text { Web of } \\
\text { science }\end{array}$ & Scopus & $\begin{array}{c}\text { Google } \\
\text { Académico }\end{array}$ \\
\hline Facilidad de lectura & $\sqrt{ }$ & $\checkmark$ & $\checkmark$ \\
\hline Adornos moderados & $\sqrt{ }$ & $\checkmark$ & $\checkmark$ \\
\hline Tipografía adecuada & $\sqrt{ }$ & $\checkmark$ & $\mathrm{X}$ \\
\hline Página funcional & $\sqrt{ }$ & $\sqrt{ }$ & $\sqrt{ }$ \\
\hline
\end{tabular}

Tabla III. Legibilidad y ergonomía.

La información que muestra la tabla IV es que las tres bases de datos permiten navegar dentro del sitio con la mayor ligereza. No importa en que parte del sitio se encuentre, con un solo clic se regresa al menú principal o bien a la sección de búsqueda.

\begin{tabular}{lccc}
\hline & $\begin{array}{c}\text { Web of } \\
\text { Science }\end{array}$ & Scopus & $\begin{array}{c}\text { Google } \\
\text { Académico }\end{array}$ \\
\hline Mapa del sitio & $\sqrt{ }$ & $\sqrt{ }$ & $\sqrt{ }$ \\
\hline $\begin{array}{l}\text { Utilización } \\
\text { de la barra } \\
\text { de desplazamiento }\end{array}$ & $\sqrt{ }$ & $\sqrt{ }$ & $\sqrt{ }$ \\
\hline $\begin{array}{l}\text { Visualización } \\
\text { de todos } \\
\text { los elementos } \\
\text { al mismo tiempo }\end{array}$ & $\sqrt{ }$ & $\sqrt{ }$ & \\
\hline $\begin{array}{l}\text { Regreso al menú } \\
\text { principal desde } \\
\text { cualquier pagina }\end{array}$ & $\sqrt{ }$ & $\sqrt{ }$ & \\
\hline $\begin{array}{l}\text { Mínimo número } \\
\text { de clics }\end{array}$ & $\sqrt{ }$ & $\sqrt{ }$ & $\sqrt{ }$ \\
\hline $\begin{array}{l}\text { Uniformidad de } \\
\text { colores }\end{array}$ & $\sqrt{ }$ & $\sqrt{ }$ & $\sqrt{ }$ \\
\hline $\begin{array}{l}\text { Claridad y unifor- } \\
\text { midad de íconos }\end{array}$ & $\sqrt{ }$ & $\sqrt{ }$ & $\sqrt{ }$ \\
\hline $\begin{array}{l}\text { Consistencia } \\
\text { en las funciones } \\
\text { de los botones } \\
\text { y etiquetas }\end{array}$ & $\sqrt{ }$ & $\sqrt{ }$ & \\
\hline
\end{tabular}

Tabla IV. Navegación y representación de la información.

\begin{tabular}{lccc}
\hline & $\begin{array}{c}\text { Web of } \\
\text { Science }\end{array}$ & Scopus & $\begin{array}{c}\text { Google } \\
\text { Académico }\end{array}$ \\
\hline Tipos de búsqueda & $\sqrt{ }$ & $\sqrt{ }$ & $\sqrt{ }$ \\
\hline Operadores boleanos & $\sqrt{ }$ & $\mathrm{X}$ & $\mathrm{X}$ \\
\hline Limitantes & $\sqrt{ }$ & $\checkmark$ & $\checkmark$ \\
\hline Índices & $\sqrt{ }$ & $\checkmark$ & $\checkmark$ \\
\hline Ayuda & $\sqrt{ }$ & $\checkmark$ & $\checkmark$ \\
\hline
\end{tabular}

Tabla V. Recuperabilidad.

Para la recuperación de información, el único criterio que no se cumple tanto en Scopus como en Google Académico es el de operadores boleanos, pues al colocar and da el mismo número de resultados. Esta información se presenta en la tabla $\mathrm{V}$.

\subsection{Macronavegación}

Hasta aquí se analizaron los aspectos de la pagina Web desde la perspectiva del mismo sitio y sobre los servicios que ofrece, pero se considera importante analizar la macronavegación que propone Codina; tres puntos que dan una panorama sobre como se ven por fuera los recursos de información.

La luminosidad (tabla $\mathrm{VI}$ ) es el número de sitios que enlazan el sitio. En este caso, a Scopus y el Web of Science, no son redireccionados por ningún sitio, porque son sitios que tienen un costo para visualizar la información y solamente las instituciones que los contratan tienen la dirección electrónica para ingresar. En cambio, Google Académico como es de libre acceso, cuanto más páginas se redireccionen mayor demanda tendrá.

\begin{tabular}{cccc}
\hline & $\begin{array}{c}\text { Web of } \\
\text { Science }\end{array}$ & Scopus & $\begin{array}{c}\text { Google } \\
\text { Académico }\end{array}$ \\
\hline $\begin{array}{c}\text { Enlaces exter- } \\
\text { nos a web }\end{array}$ & $\mathrm{X}$ & $\mathrm{X}$ & $\sqrt{ }$ \\
\hline
\end{tabular}

Tabla VI. Luminosidad.

\begin{tabular}{lccc}
\hline & $\begin{array}{c}\text { Web of } \\
\text { Science }\end{array}$ & Scopus & $\begin{array}{c}\text { Google } \\
\text { Académico }\end{array}$ \\
\hline $\begin{array}{l}\text { Calidad de la } \\
\text { información }\end{array}$ & $\sqrt{ }$ & $\sqrt{ }$ & $?$ \\
\hline
\end{tabular}

Tabla VII. Calidad de la información.

Siempre es importante evaluar la información que se da a conocer y aquí, los editores de las bases de datos si lo hacen, sobre todo Scopus y el Web of Science. La información que tiene es de calidad, ha pasado por un filtro, por lo que se garantiza la información. El Google Académico 
lo único que dice es que integra nuevas páginas, y no da a conocer los criterios para seleccionarlas o eliminarlas.

En Scopus y en el Web of Science no se ha manifestado error alguno de ligas, ni tampoco se ha interrumpido el servicio de manera drástica. Cuando lo han hecho, tienen la precaución de notificarlo con tiempo para que los usuarios tomen las debidas precauciones y no ocasione problema. En cambio en Google Académico si hay algunos sitios que no enlazan donde debieran, ocasionando molestia entre los investigadores, ya que cuando se localiza alguna información y ésta no esta disponible, no les es muy agradable (Tabla VIII).

\begin{tabular}{lccc}
\hline & $\begin{array}{c}\text { Web of } \\
\text { Science }\end{array}$ & Scopus & $\begin{array}{c}\text { Google } \\
\text { Académico }\end{array}$ \\
\hline $\begin{array}{l}\text { Actualidad de } \\
\text { los enlaces }\end{array}$ & $\sqrt{ }$ & $\sqrt{ }$ & $?$ \\
\hline
\end{tabular}

Tabla VIII. Actualización de ligas.

\subsection{Búsquedas}

La segunda parte del análisis de estas bases de datos tiene que ver con los resultados propios de una búsqueda.

Partiendo de lo general a lo particular, la primera estrategia que se realizo fue: buscar IIMAS, con sus variaciones utilizando un operado boléano y la palabra México, obteniendo por resultados los datos especificados en la tabla IX. Como se observa en esta tabla, los resultados en el Web of Science y Scopus no varían mucho, son muy similares. Sin embargo, hay que tener en cuenta es el uso de los delimitadores ya que estos, al no ser bien empleados, pueden reflejar datos no adecuados.

\begin{tabular}{lrrr}
\hline Estrategia & $\begin{array}{r}\text { Web of } \\
\text { science }\end{array}$ & Scopus & $\begin{array}{c}\text { Google } \\
\text { Académico }\end{array}$ \\
\hline iimas & 233 & 260 & 1040 \\
\hline iimas and mexico & 233 & 256 & 822 \\
\hline ins $^{*}$ inv $^{*}$ mat* appl* $^{*}$ & 209 & 8 & 34 \\
\hline $\begin{array}{l}\text { inst res appl math } \\
\text { sys }\end{array}$ & 8 & 39 & 2160 \\
\hline $\begin{array}{l}\text { univ nal aut or unam } \\
\text { and iimas }\end{array}$ & 66 & 0 & 0 \\
\hline
\end{tabular}

Tabla IX. Estrategia de búsqueda en sus diferentes variaciones usando como limitador filiación.

La tabla $\mathrm{X}$ tiene como finalidad, delimitar más los resultados de la tabla anterior, verificar que sean efectivamente resultados del IIMAS. Como se puede observar, las dos primeras bases de datos presentan variaciones muy pequeñas en el número de referencias. Esto se debe a que la información ha sido analizada y valorada con criterios bibliográficos. En cambio, Google Académico no tiene uniformidad bibliográfica; por lo tanto, al haber variaciones en los nombres de los autores, repite los artículos. Además, como no discrimina entre los tipos de documentos que hay - por ejemplo, en los resultados lo mismo puede presentar un artículo científico que uno de divulgación o una nota, o bien el currículum del autor-, la información no es académica en su totalidad.

\begin{tabular}{|c|c|c|c|}
\hline Estrategia & $\begin{array}{l}\text { Web of } \\
\text { science }\end{array}$ & Scopus & $\begin{array}{c}\text { Google } \\
\text { Académico }\end{array}$ \\
\hline iimas & 233 & 233 & 776 \\
\hline iimas and mexico & 233 & 256 & 743 \\
\hline $\begin{array}{l}\text { ins inv* mat* } \\
\text { appl }^{*}\end{array}$ & 209 & 3 & 0 \\
\hline $\begin{array}{l}\text { inst }^{*} \text { res }^{*} \text { appl } \\
\text { math* sys }\end{array}$ & 8 & 15 & 0 \\
\hline $\begin{array}{l}\text { univ nal aut or } \\
\text { unam and iimas }\end{array}$ & 66 & 0 & 0 \\
\hline
\end{tabular}

Tabla X. Búsqueda de IIMAS en sus diferentes variaciones verificando los resultados.

En el siguiente cuadro se eliminan el número de citas que se repiten quedando como resultado el número real en las tres bases de datos.

En la tabla XI se puede ver el número de citas que presenta cada base de datos solamente en lo que va de 2007, y hasta el momento 28 citas coinciden en el Web of Science y Scopus.

\begin{tabular}{lcccc}
\hline & $\begin{array}{l}\text { Web of } \\
\text { science }\end{array}$ & Scopus & $\begin{array}{c}\text { Google } \\
\text { Académico }\end{array}$ & $\begin{array}{c}\text { citas } \\
\text { repetidas }\end{array}$ \\
\hline $\begin{array}{l}\mathrm{N}^{\circ} \text { de } \\
\text { citas }\end{array}$ & 32 & 44 & 0 & 28 \\
\hline
\end{tabular}

Tabla XI. Citas localizada del IIMAS para el año 2007.

En algunas ocasiones, se solicitaron datos precisos sobre el número de citas que tienen los investigadores o el IIMAS en su conjunto, sin necesidad de contar referencia por referencia. El Web of Science facilita este dato mediante una herramienta que se llama Analyse, que proporciona en cifras el número de autores de una dependencia o bien por país y todo lo relacionado a la citas, e incluso hasta una gráfica de los resultados obtenidos. Scopus también proporciona este dato a través de su Citation tracker.

Google no proporciona datos estadísticos, por lo que obliga a realizar la búsqueda o contar los resultados obtenidos para saber cuantas citas 
tiene el IIMAS; y tampoco se puede localizar las citas por año, ya que, al incluir este campo, lo aplica en el año de publicación del artículo original. En la tabla XII se muestra el número de citas utilizando Analyse y Citation tracker.

\begin{tabular}{cccc}
\hline & $\begin{array}{c}\text { Web of } \\
\text { science }\end{array}$ & Scopus & $\begin{array}{c}\text { Google } \\
\text { Académico }\end{array}$ \\
\hline $\mathrm{N}^{\circ}$ de citas & 848 & 895 & $\mathrm{x}$ \\
\hline
\end{tabular}

Tabla XII. Número de citas que tiene el IIMAS

\begin{tabular}{lccc}
\hline \multicolumn{4}{c}{ Estrategia: Berlanga $R$ "and" año 2007 } \\
\hline & $\begin{array}{c}\text { Web of } \\
\text { Science }\end{array}$ & Scopus & $\begin{array}{c}\text { Google } \\
\text { Académico }\end{array}$ \\
\hline $\mathrm{N}^{\circ}$ de citas & 15 & 0 & 0 \\
\hline No de artículos & 4 & 0 & 2 \\
\hline $\begin{array}{l}\text { Artículos } \\
\text { pertenecientes } \\
\text { al autor }\end{array}$ & 4 & 0 & 0 \\
\hline
\end{tabular}

Tabla XIII. Estrategia de búsqueda realizada el día 12 de junio de 2007.

Para concluir con este trabajo, se realizó una estrategia de búsqueda de citas de un investigador del IIMAS. El resultado se muestra en la tabla XIII. En esta tabla los resultados son desalentadores para dos de las bases de datos, solamente el Web of Science está totalmente actualizado, ya que es el único que muestra resultados en 2007.

La tabla XIV muestra diferentes resultados al cambiar el año de la cobertura. Al ampliar el universo a los dos años anteriores, Scopus muestra información sobre las citas del autor que se esta localizando la información. Google no cuenta con una forma de buscar citas por año.

\begin{tabular}{cccc}
\hline \multicolumn{4}{c}{ Estrategia: Berlanga R año 2005-2006 } \\
\hline $\begin{array}{c}\text { Web of } \\
\text { Science }\end{array}$ & Scopus & $\begin{array}{c}\text { Google } \\
\text { Académico }\end{array}$ \\
\hline $\mathrm{N}^{\circ}$ de citas & 24 & 15 & 0 \\
\hline $\mathrm{N}^{\circ}$ de artículos & 8 & 4 & 20 \\
\hline $\begin{array}{c}\text { Artículos pertene- } \\
\text { cientes al autor }\end{array}$ & 7 & 3 & 1 \\
\hline
\end{tabular}

Tabla XIV. Estrategia de búsqueda realizada el día 12 de junio de 2007.

\section{Conclusiones}

Las citas bibliográficas están siendo consideradas por varios editores, sin embargo hay que tomar en cuenta criterios evaluativos para determinar la calidad de la información.

Por lo tanto se concluye que las tres bases de datos proporcionan información complementaria, por lo que es importante siempre verificar la duplicidad y eliminarla; y que dos bases de datos tratan la información con mayor rigor (Web of Science y Scopus). Por su parte, Google Académico no tiene estándares de calidad, ya que su objetivo es solamente conjuntar la información que se encuentra disponible en la red para darla a conocer. Scopus es un buen rival para Web of Science, aunque hay que tomar en cuenta que el Web of Science lleva muchos años en el mercado comercializando esta información y será muy difícil que tenga un competidor a su medida, más no imposible. En general, la presentación de los sitios Web es ergonómica; esto denota que han sido elaborados pensando en el usuario final, permitiendo que la navegación sea ágil y oportuna. Finalmente, este documento da una visión del comportamiento de las bases de datos. Como se mostró, Web of Science es la base de datos que tiene el liderazgo en el mercado, pero la competencia está posicionándose ya que día a día más bases de datos proporcionan citas bibliográficas.

\section{Referencias}

Codina, Lluis (2000). Evaluación de recursos digitales en línea: conceptos, indicadores y métodos. // Revista Española de Documentación Científica. 23:1. 9-44.

Golderman, Gail; Connolly, Bruce. (2007). Who Cited This? // Library Journal. 132 (Winter 2007) 18-26.

Google Académico. Googleplex, 2007. http://scholar.goo gle.com.mx / (2007-06).

Instituto de Investigaciones en Matemáticas Aplicadas y en Sistemas (2007). Informe de actividades 2006. México: El Instituto, 2007.

ISI Web of Knowledge // Scientific Thomson (2007). http://isiwebofknowledge.com / (2007-06).

LaGuardia, Cheryl (2005). ISI Web of Science/Scopus. /I Library Journal. 130 (January 2005) 40-42.

Scopus. Elsevier (2007). http://www.scopus.com/ (2007-06)

Tenopir, Carol. (2005). Google in the Academic Library. // Library Journal. 130 (January 2005) 32-32.

Web of Science. Scientific Thomson, 2007. http://isiknow ledge.com / (2007-06). 